\title{
Does EEG Montage Influence Alzheimer's Disease Electroclinic Diagnosis?
}

\author{
L. R. Trambaiolli, ${ }^{1}$ A. C. Lorena, ${ }^{1}$ F. J. Fraga, ${ }^{2}$ P. A. M. K. Kanda, ${ }^{3}$ R. Nitrini, ${ }^{3}$ and R. Anghinah ${ }^{3}$ \\ ${ }^{1}$ Mathematics, Computing and Cognition Center (CMCC), Universidade Federal do ABC (UFABC), Rua Santa Adelia, 166, \\ 09210-170 Santo Andre, SP, Brazil \\ ${ }^{2}$ Engineering, Modeling and Applied Social Sciences Center (CECS), Universidade Federal do ABC (UFABC), Rua Santa Adelia, 166, \\ 09210-170 Santo Andre, SP, Brazil \\ ${ }^{3}$ Reference Center of Behavioral Disturbances and Dementia (CEREDIC) and Neurology, Department of Medicine School of \\ University of São Paulo (USP), Rua Arruda Alvim, 206, 05.410-020 São Paulo, SP, Brazil
}

Correspondence should be addressed to F. J. Fraga, franciscojfraga@gmail.com

Received 23 December 2010; Revised 23 February 2011; Accepted 7 March 2011

Academic Editor: Fabrizio Vecchio

Copyright ( 92011 L. R. Trambaiolli et al. This is an open access article distributed under the Creative Commons Attribution License, which permits unrestricted use, distribution, and reproduction in any medium, provided the original work is properly cited.

There is not a specific Alzheimer's disease (AD) diagnostic test. AD diagnosis relies on clinical history, neuropsychological, and laboratory tests, neuroimaging and electroencephalography. Therefore, new approaches are necessary to enable earlier and more accurate diagnosis and to measure treatment results. Quantitative EEG ( $\mathrm{qEEG}$ ) can be used as a diagnostic tool in selected cases. The aim of this study was to answer if distinct electrode montages have different sensitivity when differentiating controls from $\mathrm{AD}$ patients. We analyzed EEG spectral peaks (delta, theta, alpha, beta, and gamma bands), and we compared references (Biauricular, Longitudinal bipolar, Crossed bipolar, Counterpart bipolar, and $\mathrm{Cz}$ reference). Support Vector Machines and Logistic Regression classifiers showed Counterpart bipolar montage as the most sensitive electrode combination. Our results suggest that Counterpart bipolar montage is the best choice to study EEG spectral peaks of controls versus AD.

\section{Introduction}

Alzheimer's disease $(\mathrm{AD})$ diagnosis is based upon clinical history, neuropsychological and laboratory tests, neuroimaging, and electroencephalography (EEG). New approaches are necessary to earlier and more accurate diagnosis $[1,2]$ and to measure treatment results [3].

EEG visual analysis can be a helpful diagnostic test in $\mathrm{AD}$ $[4,5]$. Background frequency displacement to delta and theta frequencies and the dropout of central alpha rhythm are common EEG findings in AD [6]. Accordingly, Sandmann et al. [7] observed a direct correlation between the degree of cognitive impairment and the power of low-frequency electrical activity in the EEG.

Since the first quantitative EEG (qEEG) studies by Lehmann [8] and Duffy et al. [9], spectral analysis (specA) and statistics have been applied to EEG. Moreover, specA has been considered from $71 \%$ to $81 \%$ sensitive to changes
[10-13] in AD EEG background. Saletu et al. [14] found a localized temporal decrease of alpha and beta activities in $\mathrm{AD}$ and slow cerebral rhythms widespread distribution in vascular dementia (VaD) [10-13]. Pucci et al. [15] proposed that a decrease in alpha frequency to $6.0-8.0 \mathrm{~Hz}$ could be an AD marker.

Despite the knowledge grounded in this field during the last decades, there are lots of unanswered questions that hinder qEEG consolidation as an AD diagnostic tool. Our objective was to study if distinct electrode montages have different sensitivity when differentiating controls from $\mathrm{AD}$ patients.

\section{Materials and Methods}

2.1. Subjects. The dataset was composed of electroencephalograms (EEGs) recorded from two groups aged from 60 to 80 years: (S1) 12 normal subjects and (S2) 22 probable $\mathrm{AD}$ patients (NINCDS-ADRDA criteria) [16]. AD group 
was classified as having mild to moderate symptoms (DSMIV-TR) [17]. Both groups were submitted to the Brazilian version of the Mini-Mental State Examination (MMSE) $[18,19]$. AD patients scored below 26 points. All probands did not have a history of diabetes mellitus, kidney disease, thyroid disease, alcoholism, liver disease, lung disease, or vitamin B12 deficiency to avoid other causes of cognitive impairment.

2.2. Data Acquisition and Processing. The EEGs were recorded with 12 bits resolution, band pass of $1-50 \mathrm{~Hz}$, and sampling rate of $200 \mathrm{~Hz}$. A Braintech 3.0 (EMSA "Equipamentos Médicos") was the recording hardware. Impedance was maintained below $10 \mathrm{~K}$, and the electrodes were placed according to the International 10-20 System [5, 20]. The interconnected ear lobe electrodes reference (without resistor) is standard in our laboratory, despite the fact that there are controversies regarding which reference is the best [21, 22]. The EEGs were recorded during 20 minutes. Probands were awake and relaxed, with closed eyes. Two skilled neurophysiologists removed EEG artifacts (blinking, drowsiness, muscle movements, or equipment-related artifacts) from the recordings. Subsequently, from each EEG, 40 epochs of eight seconds were selected by visual inspection [23].

A 512-point Hamming Fast Fourier Transform (FFT) algorithm was used to process the epochs analysis. The windows were 2.5 seconds long with $90 \%$ of overlap between successive windows [23]. EEG signals were filtered using an infinite impulse response low-pass elliptic filter with a cutoff frequency at $50 \mathrm{~Hz}$ and a zero in the frequency of $60 \mathrm{~Hz}$ to eliminate the interference of the power grid $(60 \mathrm{~Hz})$.

2.3. Feature Extraction. Feature extraction is the method used to mining some characteristics of a particular signal epoch producing data that can represent events [23]. The spectral peak feature (or peak spectrum), chosen in this work, corresponds to the frequency where the EEG spectrum amplitude reaches its maximum value. The montages used were

(i) Biauricular reference (Bar): Fp1-A1, Fp2-A2, F7-A1, F8-A2, F3-A1, F4-A2, C3-A1, C-A2, T3-A1, T4-A2, P3-A1, P4-A2, O1-A1, O2-A2;

(ii) Longitudinal Bipolar (Lbp): Fp1-F3, F3-C3, C3-P3, P3-O1, O1-T5, T5-T3, T3-F7, F7-Fp1, Fp2-F4, F4C4, C4-P4, P4-O2, O2-T6, T6-T4, T4-F9, F8-Fp2;

(iii) Crossed Bipolar (Bcr): Fp1-Fp2, F7-F3, F3-Fz, Fz-F4, F4-F8, T3-C3, C3-Cz, Cz-C4, C4-T4, T5-P3, P3-Pz, $\mathrm{Pz}-\mathrm{P} 4, \mathrm{P} 4-\mathrm{T} 6, \mathrm{O} 1-\mathrm{O} 2 ;$

(iv) Counterpart bipolar (Bco): F7-F8, F3-F4, T3-T4, C3C4, P3-P4, T5-T6, O1-O2;

(v) $\mathrm{Cz}$ reference (Czr): Fp1-Cz, Fp2-Cz, F3-Cz, F4-Cz, F7-Cz, F8-Cz, T3-Cz, T4-Cz, C3-Cz, C4-Cz, T5-Cz, T6-Cz, P3-Cz, P4-Cz, O1-Cz, O2-Cz.

Each of these electrode montages (Figure 1) had spectral peaks calculated for delta (from 0.1 to $4.0 \mathrm{~Hz}$ ), theta (from
4.0 to $8.0 \mathrm{~Hz}$ ), alpha (from 8.0 to $12.0 \mathrm{~Hz}$ ), beta (from 12.0 to $30.0 \mathrm{~Hz}$ ), and gamma (from 30.0 to $50.0 \mathrm{~Hz}$ ) bands [24].

2.4. Classifiers. The EEG dataset was composed of 1360 epochs (40 epochs of 34 subjects). The analysis was based on the leave-one-subject-out process: 1320 epochs were used for training and 40 epochs from one subject for testing. It means that, each time, the classifier was trained with epochs from all individuals except the one going to be tested. This procedure was performed to test the classifiers discriminative capacity to work with data diverse from that presented in the training period. The leave-one-subject-out process was repeated for all 34 individuals ( 34 tests each montage).

2.4.1. Support Vector Machines (SVMs). SVMs constitute a supervised Machine Learning (ML) technique based on the Statistical Learning Theory [25]. In this method, a training dataset (containing known labeled data examples) is used to draw a hyperplane with maximum margin, based on the feature coordinates, which separates the two classes (in our case, Controls and AD). Subsequently, the coordinates of this hyperplane are used to test a dataset and the accuracy of the model [26]. When classes are not linearly separable, feature coordinates should be mapped to a higher dimension by a Kernel function. In this new space, the classes become linearly separable and the maximum margin hyperplane can then be found [26].

In this experiment, the Weka tool [27] with default values was used to the SVM induction. The regularization coefficient of SVM was maintained in $C=1.0$, while the Kernel used was RBF [28]. The cache size was 250007, and the gamma value was 0.01 .

2.4.2. Logistic Regression (LR). Logistic regression is part of a category of statistical models called generalized linear models. LR is a classification tool frequently used to help diagnosis [29]. In this method, the discriminant function analyses the sum of the scores of each feature and then delimitates the boundaries between the two groups [30]. Logistic regression calculates the predicted probability of different subgroups (in our analysis) falling into a category [30]. In LR induction, we also used Weka tool [27] with default values. In this case, the maximum interaction value was -1.0 , and the ridge value in the log-likelihood was configured to 1.0 .

\section{Results and Discussion}

Table 1 shows the results of both classifiers to each electrode montage. The columns represent, respectively, from left to right, accuracy, sensitivity (patients correctly diagnosed as $\mathrm{AD}$ ), and specificity (controls correctly diagnosed as normals). The first line of each montage shows the percentage of epoch classification (mean and standard deviations).

The second line of Table 1 presents the per subject percentage. The leave-one-out analysis of each subject took into consideration the ratio between the number of epochs classified correctly and the total number of epochs. When this 


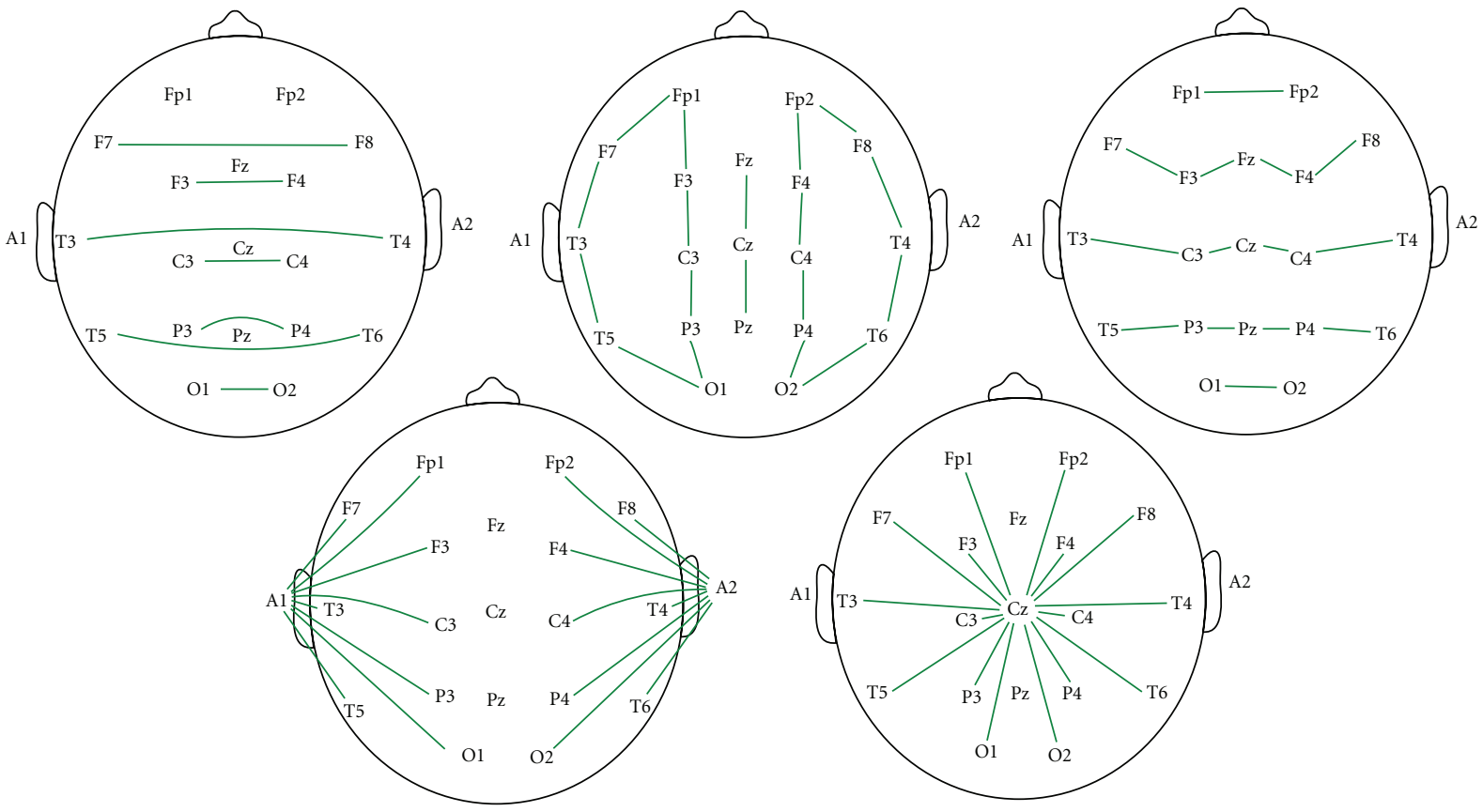

FIGURE 1: Spectral peaks montage maps. Lines correspond to subtractions used to calculate spectral peaks. From left to right, top to bottom: Counterpart Bipolar (Bco), Longitudinal Bipolar (Lbp), Crossed Bipolar (Bcr), Biauricular reference (Bar), and Cz reference (Czr).

TABLe 1: Accuracy, sensitivity, and specificity rates for each montage. Best results in bold and worst results in italic.

\begin{tabular}{|c|c|c|c|c|c|c|}
\hline & \multicolumn{3}{|c|}{ Support Vector Machines } & \multicolumn{3}{|c|}{ Logistic Regression } \\
\hline & Accuracy (\%) & Sensitivity (\%) & Specificity (\%) & Accuracy (\%) & Sensitivity (\%) & Specificity (\%) \\
\hline & \multicolumn{3}{|c|}{ Bipolar Counterpart } & \multicolumn{3}{|c|}{ Bipolar Counterpart } \\
\hline Epochs & $81,32 \pm 28,00$ & $89,43 \pm 20,92$ & $66,46 \pm 33,84$ & $82,13 \pm 20,86$ & $86,93 \pm 17,49$ & $73,33 \pm 24,32$ \\
\hline \multirow[t]{2}{*}{ Patient } & 85,29 & 90,91 & 75,00 & 91,18 & 95,45 & 83,33 \\
\hline & \multicolumn{3}{|c|}{ Longitudinal Bipolar } & \multicolumn{3}{|c|}{ Longitudinal Bipolar } \\
\hline Epochs & $72,72 \pm 36,80$ & $84,09 \pm 27,52$ & $51,88 \pm 43,39$ & $66,03 \pm 35,76$ & $75,45 \pm 31,42$ & $48,75 \pm 38,04$ \\
\hline \multirow[t]{2}{*}{ Patient } & 79,41 & 90,91 & 58,33 & 64,71 & 72,73 & 50,00 \\
\hline & \multicolumn{3}{|c|}{ Crossed Bipolar } & \multicolumn{3}{|c|}{ Crossed Bipolar } \\
\hline Epochs & $69,19 \pm 37,60$ & $80,23 \pm 32,40$ & $48,96 \pm 39,32$ & $65,07 \pm 36,09$ & $76,59 \pm 33,23$ & $43,96 \pm 32,36$ \\
\hline \multirow[t]{2}{*}{ Patient } & 64,71 & 81,82 & 41,67 & 67,65 & 77,27 & 50,00 \\
\hline & \multicolumn{3}{|c|}{ Biauricular Reference } & \multicolumn{3}{|c|}{ Biauricular Reference } \\
\hline Epochs & $70,07 \pm 36,81$ & $85,57 \pm 23,12$ & $41,67 \pm 41,03$ & $66,32 \pm 32,50$ & $76,14 \pm 28,05$ & $48,33 \pm 33,50$ \\
\hline \multirow[t]{2}{*}{ Patient } & 76,47 & 95,45 & 41,67 & 67,65 & 81,82 & 41,67 \\
\hline & \multicolumn{3}{|c|}{ Cz Reference } & \multicolumn{3}{|c|}{$\mathrm{Cz}$ Reference } \\
\hline Epochs & $70,22 \pm 37,70$ & $81,36 \pm 31,15$ & $49,79 \pm 41,33$ & $71,62 \pm 28,37$ & $80,45 \pm 24,64$ & $55,42 \pm 28,52$ \\
\hline Patient & 70,59 & 81,82 & 50,00 & 73,53 & 86,36 & 50,00 \\
\hline
\end{tabular}

ratio was over 0.5 , the subject classification was considered correct. After 34 tests, the rate of subject correct diagnosis was calculated. In Table $1, \mathrm{Bco}$ is the montage with highest number of correct diagnosis and the lowest standard deviation to all classifiers. Bco also had high specificity (correct diagnosis of $\mathrm{AD}$ ) and sensibility. These findings are relevant because they validate this qEEG technique as a diagnostic method. Therefore, it can help supporting clinical diagnosis.

It is important to note that high standard deviation (SD) is a methodological consequence of the leave-one-subjectout test. If an individual had bad epochs accuracy, the group mean was low and the SD high. Bco was the montage with 
TABle 2: Number of patients with epoch accuracy rates equal to $100 \%$, exceeding or equal to $75 \%$, less than or equal to $50 \%$, and equal to $0 \%$ for each test. Best results in bold and worst results in italic.

\begin{tabular}{|c|c|c|c|c|c|c|c|c|}
\hline & \multicolumn{4}{|c|}{ Support Vector Machines } & \multicolumn{4}{|c|}{ Logistic Regression } \\
\hline & $=100$ & $\geq 75$ & $\leq 50$ & $=0$ & $=100$ & $\geq 75$ & $\leq 50$ & $=0$ \\
\hline Bipolar Counterpart & 15 & 26 & 5 & $\mathbf{0}$ & 12 & 25 & 3 & $\mathbf{0}$ \\
\hline Longitudinal Bipolar & 16 & 21 & 7 & 2 & 7 & 20 & 12 & 1 \\
\hline Crossed Bipolar & 13 & 20 & 12 & 3 & 8 & 18 & 11 & 2 \\
\hline Biauricular Reference & 14 & 19 & 8 & 2 & 8 & 18 & 11 & 0 \\
\hline $\mathrm{Cz}$ Reference & 15 & 20 & 10 & 3 & 4 & 20 & 9 & $\mathbf{0}$ \\
\hline
\end{tabular}

TABLE 3: Odds ratio to Bipolar Counterpart LR test. In bold the significant ones $(>1)$.

\begin{tabular}{lcccrr}
\hline & delta & theta & alpha & beta & gamma \\
\hline F3-F4 & $0,371 \pm 0,063$ & $\mathbf{2 , 4 9 6} \pm \mathbf{0 , 5 7 5}$ & $0,969 \pm 0,230$ & $\mathbf{1 , 1 8 8} \pm \mathbf{0 , 1 0 1}$ & $0,913 \pm 0,052$ \\
F7-F8 & $\mathbf{1 2 8 , 8 0 6} \pm \mathbf{5 0 , 8 0 6}$ & $\mathbf{2 , 5 8 0} \pm \mathbf{0 , 9 5 8}$ & $0,728 \pm 0,221$ & $\mathbf{1 , 5 4 3} \pm \mathbf{0 , 1 2 6}$ & $0,659 \pm 0,045$ \\
C3-C4 & $0,693 \pm 0,176$ & $\mathbf{3 , 6 6 7} \pm \mathbf{1 , 2 1 3}$ & $0,734 \pm 0,191$ & $\mathbf{2 , 2 2 9} \pm \mathbf{0 , 2 7 5}$ & $0,975 \pm 0,047$ \\
T3-T4 & $0,753 \pm 0,182$ & $0,177 \pm 0,068$ & $0,263 \pm 0,059$ & $0,836 \pm 0,087$ & $\mathbf{1 , 1 1 8} \pm \mathbf{0 , 0 4 9}$ \\
T5-T6 & $0,277 \pm 0,075$ & $\mathbf{1 , 0 1 1} \pm \mathbf{0 , 2 5 8}$ & $0,104 \pm 0,029$ & $0,875 \pm 0,080$ & $0,649 \pm 0,034$ \\
P3-P4 & $0,574 \pm 0,107$ & $0,511 \pm 0,091$ & $0,019 \pm 0,008$ & $0,962 \pm 0,118$ & $\mathbf{1 , 8 8 8} \pm \mathbf{0 , 0 8 7}$ \\
O1-O2 & $\mathbf{2 , 2 3 1} \pm \mathbf{0 . 3 6 4}$ & $0,402 \pm 0,126$ & $0,767 \pm 0,177$ & $0,761 \pm 0,072$ & $0,792 \pm 0,036$ \\
\hline
\end{tabular}

lower SD, consequently, indicating less variability in number of correct diagnosis.

Table 2 shows the results of the individual accuracy rate variability. The columns show, respectively, from left to right, epochs accuracy by each subject of $100 \%, \geq 75 \%, \leq 50 \%$, and $0 \%$ (all epochs incorrectly classified by one subject).

SVMs tests presented Lbp as the montage with maximum epoch accuracy (16 subjects with 100\% accuracy), followed by Bcp e Czr (15 cases each). Bco was the montage with higher number of cases with accuracy greater than or equal to $75 \%$, less cases with accuracy less than or equal to $50 \%$, and without cases of $0 \%$ correct classification.

The LR tests ratified Bco as having the highest number of $100 \%$ accuracy results, the highest number of cases with accuracy greater than or equal to $75 \%$, less cases with accuracy less than $50 \%$, and no cases of $0 \%$ correct classification (in this last case similar to Bar and Czr, both with zero cases).

This study suggested that Bco was the more trustworthy montage because of his higher rates of $100 \%$ epoch accuracy and absence of $0 \%$ cases to both classifiers. Consequently, other parameters could be tested based on LR. The odds ratio values (ODDR) could be analyzed from the ratio $\mathrm{AD} /$ controls (Table 3 ). It was possible to verify 11 features presenting ODDR $>1$. Consequently, there is a possibility that these features can be associated with $\mathrm{AD}$.

Among these ODDR features, the electrodes F3-F4, F7F8, C3-C4, and T5-T6 presented values of ODDR $>1$ to theta band; the electrodes F7-F8 and O1-O2 presented values of ODDR > 1 to delta band; F3-F4, F7-F8 and C3-C4 presented values of ODDR > 1 to beta band, and T3-T4 and P3-P4 presented values of ODDR $>1$ to gamma band.

EEGs of mild DA have higher theta activity and low beta activity $[31,32]$, as seen in our cases (F3-F4, F7-F8, C3C4, and T5-T6). Furthermore, these electrodes were directly associated with the inter-hemispheric differences found in our AD population [33]. Moderate to advanced cases of AD are associated with increasing of delta activity [32, 34-36], and this could explain the values found in F7-F8 and O1$\mathrm{O} 2$. Thus, our findings are in accordance to data presented by others.

The analysis of the number of electrodes related to each montage demonstrates that the montages with higher number of signals were Lbp and Czr with 16 signals each, followed by Bar and Bcr with 14 signals. The montage with lowest number of signals was Bco (7 signals). We can say that $\mathrm{Bco}$ is also the more compact (less electrodes), consequently, less expensive in terms of processing time.

\section{Conclusion}

To sum, our results are in accordance with the literature that suggests that the spectral peak is an efficient tool in $\mathrm{AD}$ diagnosis $[24,37]$. Our contribution is to answer the question that gave origin to the paper. Yes, the analysis indicates that the bipolar inter-hemispheric montage (Counterpart bipolar) is the best to evaluate $\mathrm{AD}$ patients with the help of automatic classifiers (DA versus N) $[38,39]$, when using EEG spectral peaks as features (predictors). 
Although more tests are needed to confirm the generalization power of our classifiers, we propose that spectral peak calculation using different montages of electrodes have an influence on the classification results (differentiation) of normal subjects and patients with $\mathrm{AD}$. Our future goal is to generalize the results obtained increasing the number of probands.

\section{References}

[1] H. Adeli, S. Ghosh-Dastidar, and N. Dadmehr, "Alzheimer's disease: models of computation and analysis of EEGs," Clinical EEG and Neuroscience, vol. 36, no. 3, pp. 131-140, 2005.

[2] A. Martínez, D. K. Lahiri, E. Giacobini, and N. H. Greig, "Advances in alzheimer therapy: understanding pharmacological approaches to the disease," Current Alzheimer Research, vol. 6, no. 2, pp. 83-85, 2009.

[3] R. Sneddon, W. R. Shankle, J. Hara, A. Rodriquez, D. Hoffman, and U. Saha, "qEEG monitoring of Alzheimer's disease treatment: a preliminary report of three case studies," Clinical EEG and Neuroscience, vol. 37, no. 1, pp. 54-59, 2006.

[4] M. R. Nuwer, G. Comi, R. Emerson et al., "IFCN standards for digital recording of clinical EEG," Electroencephalography and Clinical Neurophysiology, vol. 106, no. 3, pp. 259-261, 1998.

[5] F. J. C. Luccas, R. Anghinah, N. I. O. Braga et al., "Guidelines for recording/analyzing quantitative EEG and evoked potentials: part II. Clinical aspects," Arquivos de Neuro-Psiquiatria, vol. 57, no. 1, pp. 132-146, 1999.

[6] D. W. Klass and R. P. Brenner, "Electroencephalography of the elderly," Journal of Clinical Neurophysiology, vol. 12, no. 2, pp. 116-131, 1995.

[7] M. C. Sandmann, E. R. Piana, D. S. Sousa, and P. R. M. De Bittencourt, "Digital EEG with brain mapping in Alzheimer's dementia and Parkinson's disease: a prospective controlled study," Arquivos de Neuro-Psiquiatria, vol. 54, no. 1, pp. 50-56, 1996.

[8] D. Lehmann, "Multichannel topography of human alpha EEG fields," Electroencephalography and Clinical Neurophysiology, vol. 31, no. 5, pp. 439-449, 1971.

[9] F. H. Duffy, J. L. Burchfiel, and C. T. Lombroso, "Brain electrical activity mapping (BEAM): a method for extending the clinical utility of EEG and evoked potential data," Annals of Neurology, vol. 5, no. 4, pp. 309-321, 1979.

[10] M. Martin-Loeches, P. Gil, F. Jimenez et al., "Topographic maps of brain electrical activity in primary degenerative dementia of the Alzheimer type and multiinfarct dementia," Biological Psychiatry, vol. 29, no. 3, pp. 211-223, 1991.

[11] T. Dierks, I. Perisic, L. Frolich, R. Ihl, and K. Maurer, “Topography of the quantitative electroencephalogram in dementia of the Alzheimer type: relation to severity of dementia," Psychiatry Research, vol. 40, no. 3, pp. 181-194, 1991.

[12] A. F. Leuchter, I. A. Cook, T. F. Newton et al., "Regional differences in brain electrical activity in dementia: use of spectral power and spectral ratio measures," Electroencephalography and Clinical Neurophysiology, vol. 87, no. 6, pp. 385-393, 1993.

[13] A. F. Leuchter, J. E. Spar, D. O. Walter, and H. Weiner, "Electroencephalographic spectra and coherence in the diagnosis of Alzheimer's-type and multi-infarct dementia. A pilot study," Archives of General Psychiatry, vol. 44, no. 11, pp. 993-998, 1987.

[14] B. Saletu, P. Anderer, and H. V. Semlitsch, "Relations between symptomatology and brain function in dementias: doubleblind, placebo-controlled, clinical and EEG/ERP mapping studies with nicergoline," Dementia and Geriatric Cognitive Disorders, vol. 8, supplement 1, pp. 12-21, 1997.

[15] E. Pucci, N. Belardinelli, G. Cacchiò, M. Signorino, and F. Angeleri, "EEG power spectrum differences in early and late onset forms of Alzheimer's disease," Clinical Neurophysiology, vol. 110, no. 4, pp. 621-631, 1999.

[16] G. McKhann, D. Drachman, and M. Folstein, "Clinical diagnosis of Alzheimer's disease: report of the NINCDS-ADRDA work group under the auspices of Department of Health and Human Services Task Force on Alzheimer's disease," Neurology, vol. 34, no. 7, pp. 939-944, 1984.

[17] American Psychiatric Association, Diagnostic and Statistical Manual (DSM-IV-TR), American Psychiatric Association, 3rd edition, 2000.

[18] M. F. Folstein, S. E. Folstein, and P. R. McHugh, "Mini mental state'. A practical method for grading the cognitive state of patients for the clinician," Journal of Psychiatric Research, vol. 12, no. 3, pp. 189-198, 1975.

[19] S. M. D. Brucki, R. Nitrin, P. Caramelli, P. H. F. Bertolucci, and I. H. Okamoto, "Suggestions for utilization of the mini-mental state examination in Brazil," Arquivos de Neuro-Psiquiatria, vol. 61, no. 3B, pp. 777-781, 2003.

[20] G. H. Klem, H. O. Lüders, H. H. Jasper, and C. Elger, "The ten-twenty electrode system of the International Federation. The International Federation of Clinical Neurophysiology," Electroencephalography and Clinical Neurophysiology, vol. 52, pp. 3-6, 1999.

[21] P. Rappelsberger, "The reference problem and mapping of coherence: a simulation study," Brain Topography, vol. 2, no. 1-2, pp. 63-72, 1989.

[22] D. Yao, L. Wang, L. Arendt-Nielsen, and A. C. Chen, "The effect of reference choices on the spatio-temporal analysis of brain evoked potentials: the use of infinite reference," Computers in Biology and Medicine, vol. 37, no. 11, pp. 15291538, 2007.

[23] S. Sanei and J. Chambers, EEG Signal Processing, WileyInterscience, New York, NY, USA, 2007.

[24] R. Anghinah, Estudo da densidade espectral e da coerência do eletroencefalograma em indivíduos normais e com doença de Alzheimer provável, Ph.D. thesis, Faculdade de Medicina da Universidade de São Paulo, São Paulo, Brazil, 2003.

[25] V. N. Vapnik, The Nature of Statistical Learning Theory, Springer, New York, NY, USA, 1995.

[26] N. Cristianini and J. Shawe-Taylor, An Introduction to Support Vector Machines: and Other Kernel-Based Learning Methods, Cambridge University Press, Cambridge, UK, 2000.

[27] I. A. Witten and E. Frank, Data Mining: Practical Machine Learning Tools and Techniques, Elsevier, 2005.

[28] L. R. Trambaiolli, A. C. Lorena, F. J. Fraga, and R. Anghinah, "Uso de aprendizado de máquina no auxílio ao diagnóstico de Alzheimer," REIC-Revista Eletrônica de Iniciação Científica, vol. 9, no. 3, pp. 1-14, 2009 (Portuguese).

[29] D. Pregibon, "Logistic regression diagnostics," The Annals of Statistics, vol. 9, no. 4, pp. 705-724, 1981.

[30] D. Hosmer and S. Lemeshow, Applied Logistic Regression, Wiley-Interscience, New York, NY, USA, 2000.

[31] L. A. Coben, W. L. Danziger, and L. Berg, "Frequency analysis of the resting awake EEG in mild senile dementia of Alzheimer type," Electroencephalography and Clinical Neurophysiology, vol. 55, no. 4, pp. 372-380, 1983.

[32] L. A. Coben, W. Danziger, and M. Storandt, "A longitudinal EEG study of mild senile dementia of Alzheimer type: changes at 1 year and at 2.5 years," Electroencephalography and Clinical Neurophysiology, vol. 61, no. 2, pp. 101-112, 1985. 
[33] F. H. Duffy, M. S. Albert, and G. McAnulty, "Brain electrical activity in patients with presenile and senile dementia of the Alzheimer type," Annals of Neurology, vol. 16, no. 4, pp. 439448, 1984.

[34] D. B. Hier, C. A. Mangone, R. Ganellen et al., "Quantitative measurement of delta activity in Alzheimer's disease," Clinical EEG Electroencephalography, vol. 22, no. 3, pp. 178-182, 1991.

[35] M. Penttilä, J. V. Partanen, H. Soininen, and P. J. Riekkinen, "Quantitative analysis of occipital EEG in different stages of Alzheimer's disease," Electroencephalography and Clinical Neurophysiology, vol. 60, no. 1, pp. 1-6, 1985.

[36] B. Stigsby, G. Johannesson, and D. H. Ingvar, "Regional EEG analysis and regional cerebral blood flow in Alzheimer's and Pick's diseases," Electroencephalography and Clinical Neurophysiology, vol. 51, no. 5, pp. 537-547, 1981.

[37] I. Raicher, D. Takahashi, P. Kanda, R. Nitrini, and R. Anghinah, "qEEG spectral peak in Alzheimer's disease," Dementia and Neuropsychologia, vol. 2, no. 1, pp. 9-12, 2008.

[38] L. R. Trambaiolli, A. C. Lorena, F. J. Fraga, and R. Anghinah, "Support vector machines in the diagnosis of Alzheimer's disease," in Proceedings of the ISSNIP Biosignals and Biorobotics Conference, vol. 1, pp. 1-6, 2010.

[39] Y. T. Kwak, "Quantitative EEG findings in different stages of Alzheimer's disease," Journal of Clinical Neurophysiologyy, vol. 23, no. 5, pp. 456-461, 2006. 


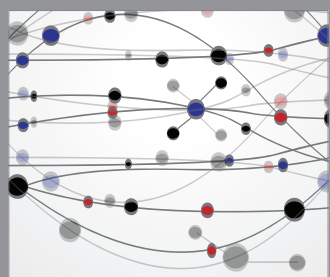

The Scientific World Journal
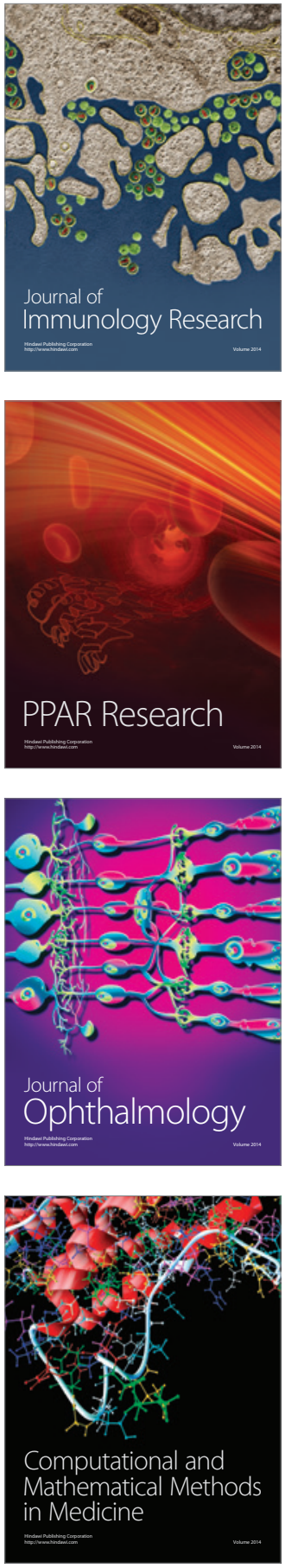

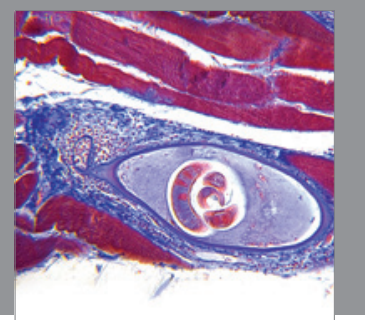

Gastroenterology

Research and Practice
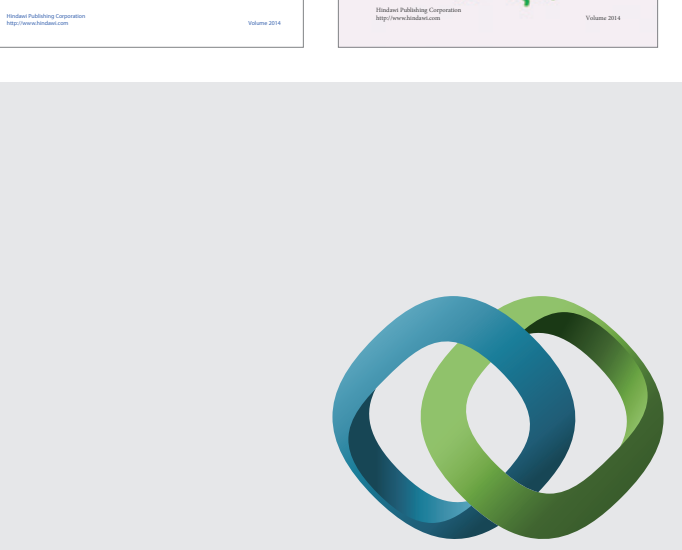

\section{Hindawi}

Submit your manuscripts at

http://www.hindawi.com
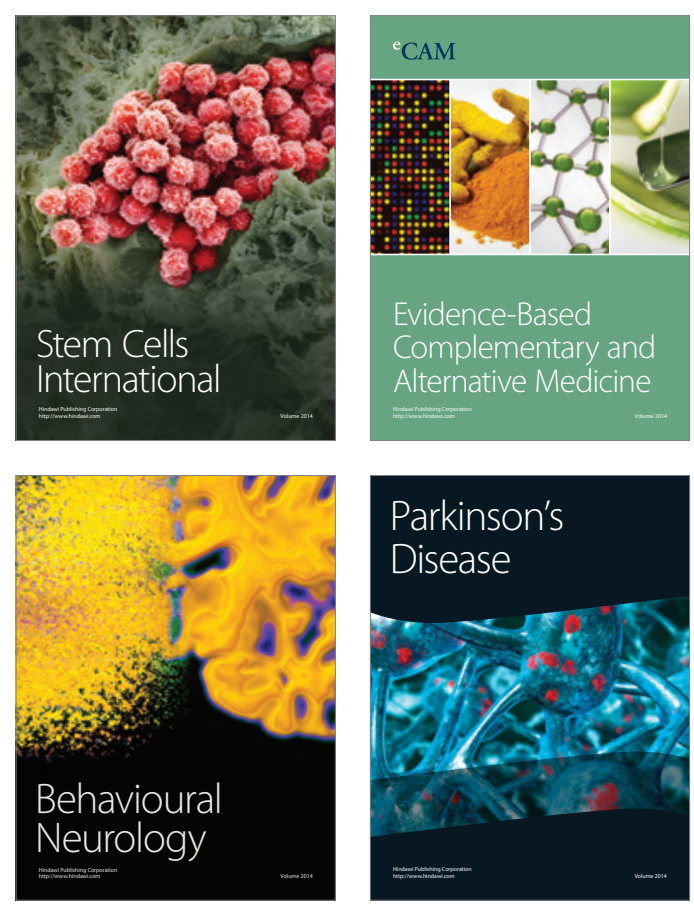

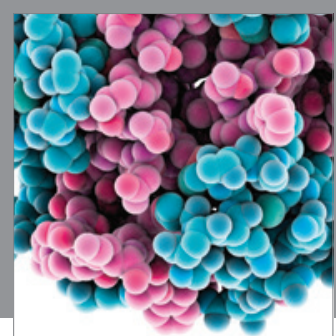

Journal of
Diabetes Research

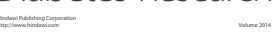

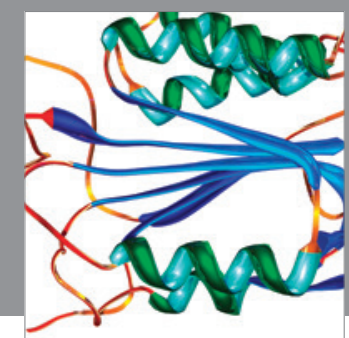

Disease Markers
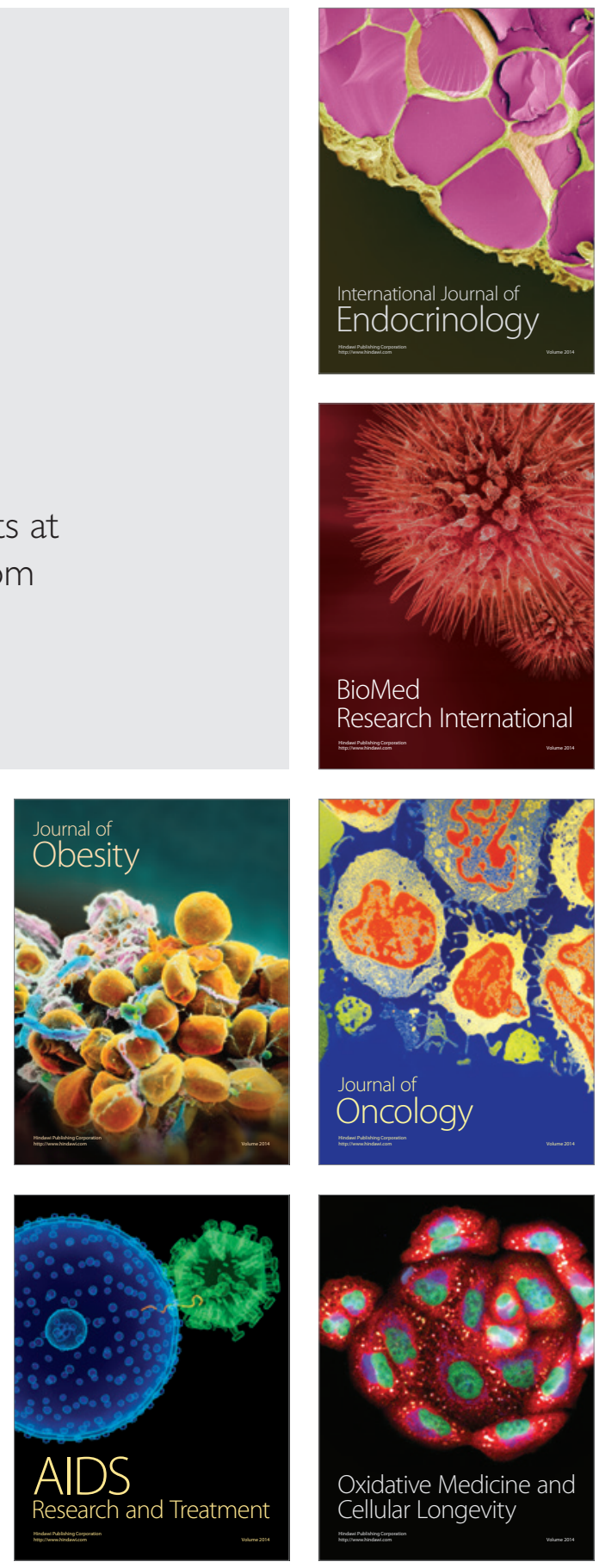